\title{
A new oxidative derivatization method for spectrophotometric determination of Periciazine in pharmaceutical preparations
}

\author{
Mykola Ye. Blazheyevskiy*, Valeriy P. Moroz ${ }^{\mathrm{b}}$ \\ a Physical and Colloid Chemistry Department, National University of Pharmacy, 53 Pushkins'ka \\ str., Kharkiv, 61002, Ukraine \\ b Analytical Chemistry Department, National University of Pharmacy, 53 Pushkins'ka str., Kharkiv, \\ 61002, Ukraine \\ blazejowski@ukr.net
}

Keywords: Spectrophotometry, oxone, Periciazine, phenothiazine sulphoxide, pharmaceutical preparations

A new the oxidative derivatization method by means of peroxoacid for the indirect spectrophotometric determination of Periciazine is presented. A potassium hydrogenperoxymonosulfateas a derivatizing agent for Periciazine, yielding the absorbative Periciazine sulfoxide at $\lambda_{\max }=362 \mathrm{~nm}$ is proposed. This reaction product was successfully employed for spectrophotometric determination of the Periciazine. The UV spectrophotometric determination of the Periciazine as its sulfoxide proved to be the more simple and selective method. Limit of quantification ( $\mathrm{LOQ}=10 \mathrm{~S}$ ) is $2.8 \mu \mathrm{g} \cdot \mathrm{mL}^{-1}$. The common excipients employed do not interfere in the determination of phenothiazine drug. Results of analysis of the drug dosage forms by the proposed method are in good agreement with those of the official method. RSD $=1.76 \%(\delta<\mathrm{RSD})$.

\section{Introduction}

Periciazine (Synonyms: Pericyazine, ProPericiazine (PCZ), Neuleptil, Neulactil,) chemically, 3-cyano-10 (3-4'-hydroxy piperidinopropyl) phenothiazine or 10-[3-(4Hydroxypiperidino)propyl]phenothiazine-2carbonitrile; 1-[3-(2-Cyanophenothiazin-10yl)propyl]piperidin-4-ol; (Figure 1)is usually given as the base but the mesilate and tartrate have also been used; a synthetic piperidine phenothiazine derivative with general properties similar to those of chlorpromazine. It is used in the treatment of psychoses including schizophrenia and disturbed behavior, and in the short-term management of severe anxiety $[1,2]$. Periciazine is a phenothiazine derivative used for the treatment of people with schizophrenia and is reputed to have a low level of extrapyramidal adverse effects.

Very few analytical methods have been reported with Periciazine individually and along with other drugs. Among that there is 
spectrophotometric method based on the development of red colored products by the interaction of phenothiazines with diazotisedanthranilic acid in hydrochloric acid medium.<smiles>N#Cc1ccc2c(c1)N(CCCN1CCC(O)CC1)c1ccccc1S2</smiles>

Figure 1. Chemical structure of Periciazine base

The reaction proceeds via the oxidation of the phenothiazine nucleus into a semiquinonoid radical [3].Flow-injection procedure of the spectrophotometric determination of Periciazine based on its the oxidation reaction by means of $\mathrm{Fe}(\mathrm{III})$ and $\mathrm{K}_{3}\left[\mathrm{Fe}(\mathrm{CN})_{6}\right.$ [4], differential pulse and square wave voltammetric methods [5] and refractometry [6] have beenalready reported. Several chromatographic methods have been published for the determination of Periciazine in bulk or in different pharmaceutical formulations as in biological fluids as well. A highperformance liquidchromatographic method (HPLC) has been developed for the simultaneous analysis of the Periciazine and another phenothiazines in human serum using spectrophotometric detection [7]. A highly sensitive liquid chromatography-tandem mass spectrometry method was developed for the determination of Periciazine in the presence of 7hydroxy and sulphoxide metabolites of Periciazine in human plasma after liquid-liquid extraction with ethyl acetate [8]. The chromatographic behavior of phenothiazine derivatives was studied by thin-layer chromatography with the use of Sorbfil silica gel plates in a binary benzene-methanol mixture of solvents [9]. A chromatographic conditionswere optimized and thin-layer chromatography method for determination of phenothiazine derivatives was described. Chromatographic systems with different polarity eluents in the wide composition range for screening of phenothiazine derived was studied [10].

The official compendia USP-39 [11] and $\mathrm{Ph}$ Eur. 9 [12] for the determination of phenothiazines in bulk, or in pharmaceutical formulations involves measurements of the absorbance at selected wavelengths, potentiometric titration in a non-aqueous medium and also HPLC method for estimation of API content in pharmaceutical preparations.

HPLC technique, though selective and sensitive, is relatively expensive involving costly instruments and solvents.

The main disadvantage of direct spectrophotometry in UV region is the sensitivity to excipients usually presented in pharmaceutical preparations. The descriptions given in the presented review methods, based on their oxidation reaction, can be alternatives. The absorbance of phenothiazine sulphoxides is less liable to spectral interferences from others ingredients of pharmaceuticals [13]. The described methods offer advantages in their simplicity, rapidity and common access to 
instrumentation. There are only a few methods based on the UV-absorption spectra of phenothiazines for their determination (e.g. on the absorption measurements in the methanol solution). The methods may be recommended as alternatives to the official methods.

The purpose of the work was to develop a new analytical method for the quantitative determination of Periciazine in dosage forms in the presence of inactive ingredients by indirect absorption optical spectrophotometry in the form of a respective sulfoxide produced with potassium hydrogen peroxymonosulfate as an oxidant.

Based on the results of the comparative study of the electron spectra of light absorption of solutions of Periciazine and its S-oxidation product with hydrogen peroxymonosulfate in acidic media, we proposed a selective method for spectrophotometric determination of Periciazine in $10 \mathrm{mg}$ capsules and in a $4 \%$ solution (drops) for intravenous use.

\section{Experimental part}

\section{Apparatus}

Registration of spectra of Periciazine solutions and products of its chemical oxidation, as well as measurement of absorbance of solutions, were carried out in a $1 \mathrm{~cm}$ thick quartz cuvette on an Evolution 60S UV-Visible Thermo-Scientific Spectrophotometer (USA) against a solution without the studied phenothiazine derivative or double-distilled water (compensation solution).

The subject of the test was the finished form of the well-known drug Neuleptil ${ }^{\circledR}, 10 \mathrm{mg}$ capsules No. 5, manufactured by SANOFI Famarella Chea Services Madrid S.A.U., Spain, number 17N0020 series. One capsule of Neuleptil contains $10 \mathrm{mg}$ of Periciazine active ingredient, as well as inactive ingredients such as magnesium stearate $(3 \mathrm{mg})$ and calcium hydrogen phosphate dihydrate $(137 \mathrm{mg})$. As part of the capsule itself, there are such chemicals as gelatin and titanium dioxide. According to the analysis certificate, the average content of the drug (Periciazine base) was $10.07 \mathrm{mg}$ in one capsule (limits - not less than 9.50 and not more than $10.50 \mathrm{mg}$ to one capsule, that is $95-105 \%$ ).

Neuleptil, a $30 \mathrm{~mL} 4 \%$ oral (solution) drops containing $4 \mathrm{~g}$ of the Periciazine active ingredient, and also inactive ingredients such as purified water $(100 \mathrm{~mL})$, glycerol $(15 \mathrm{~g})$, ascorbic acid $(0.8 \mathrm{~g})$, ether oil, peppermint leaf extract (0.04 g), saccharose (sucrose) (25 g) and E150d (caramel, $0.2 \mathrm{~g}$ ), tartaric acid (1.65 g) and 96\% ethanol $(9.74 \mathrm{~g})$. SANOFI - AVENTIS FRANCE (France), produced by A. Hutterman\&Sie, GmbH, Germany.

According to the Certificate of Analysis (series No. 6K0331), the average content of the drug (Periciazine active substance) was $3.96 \%$ in one capsule (limits of not less than 3.8 and not more than $4.2 \%$, that is $95-105 \%$ ).

\section{Reagents}


In this experiment, the oxidation of a Periciazine to a S-oxide Periciazine using a potassium triple salt containing potassium peroxymonosulfate $\left(\mathrm{KHSO}_{5}\right)$, potassium hydrogensulfate $\left(\mathrm{KHSO}_{4}\right)$, and potassium sulfate $\left(\mathrm{K}_{2} \mathrm{SO}_{4}\right)$ in a 2:1:1 molar ratio was realized. This product is sold under the trade name Oxone ${ }^{\circledR}$ Its formula weight is 614.8 . Moreover, it is considered as "green" oxidizing agent because it has not toxic effects.

Preparation of $0.005 \mathrm{~mol} \mathrm{~L}^{-1}$ potassium hydrogen peroxymonosulfate solution. About 0.15-0.2 g of Oxone ${ }^{\circledR}$ was dissolved in $100 \mathrm{~mL}$ of double-distilled water. The content of potassium hydrogen peroxymonosulfate was determined by iodometric titration.

Working Standard solution (WSS) of

\section{Periciazine}

The working standard Periciazine solution, $0.10 \mathrm{mgmL}^{-1}$, is prepared by volumeweight method. A weighted amount of substance powder with a known content of the active substance containing $10.0 \mathrm{mg}$ of Periciazine, recalculation on the Periciazine base $\left(\mathrm{C}_{21} \mathrm{H}_{23} \mathrm{~N}_{3} \mathrm{OS}\right)$, is dissolved in $100.0 \mathrm{~mL}$ of a $0.02 \mathrm{~mol} \mathrm{~L}-1$ hydrochloric acid solution at $+20^{\circ} \mathrm{C}$.

\section{Procedures}

\section{Preparation of calibration graph}

An accurately measured by means of microburette, aliquot volumes of $2.5 ; 5 ; 10 ; 15$; $20 \mathrm{~mL}$ of Periciazine WSS are transfered in 50 $\mathrm{mL}$ volumetric flasks. After that, $5 \mathrm{~mL}$ of $0.2 \mathrm{~mol}$ $\mathrm{L}^{-1}$ hydrochloric acid and $20 \mathrm{~mL}$ of a $0.005 \mathrm{~mol}$
$\mathrm{L}^{-1}$ potassium hydrogen peroxymonosulfate solution were successively added. Then, it was necessary to shake thoroughly and bring the volume of the solution to the mark with doubledistilled water and close the flask and mix thoroughly. The solutions are measured at an analytical wavelength of $362 \mathrm{~nm}$. Doubledistilled water as a compensating solution was used.

\section{Procedure for Pharmaceutical}

\section{Preparations}

Method for Periciazine content determination in $10 \mathrm{mg}$ capsules. An accurately weighed amount of about $0.150 \mathrm{~g}$ of powder containing capsules $(10 \mathrm{mg})$ corresponding to the average weight of the capsule is mixed with $50 \mathrm{~mL}$ of a $0.2 \mathrm{~mol} \mathrm{~L}^{-1}$ solution of hydrochloric acid and shaken thoroughly for $30 \mathrm{~min}$, filtered through a "yellow tape" filter, the residue is thoroughly rinsed on filter ( 3 times, $10 \mathrm{~mL}$ ) with double-distilled water. Combine the filtrate, quantitatively transfer the solution to a $100 \mathrm{~mL}$ volumetric flask. The volume of the solution to the mark is adjusted with double-distilled water and mixed thoroughly. Take $20 \mathrm{~mL}$ of the Periciazine solution obtained by pipette and transfer to a $50 \mathrm{~mL}$ volumetric flask, add $5 \mathrm{~mL}$ of a $0.2 \mathrm{~mol} \mathrm{~L}^{-1}$ solution of hydrochloric acid and $20.0 \mathrm{~mL}$ of $0.005 \mathrm{~mol} \mathrm{~L}^{-1}$ potassium hydrogen peroxymonosulfate solution, carefully shake and bring the solution to the mark with doubledistilled water, close the flask and mix thoroughly 
Similar procedures wereperformed with a standard solution was carried out.In this case, a volume of $20 \mathrm{~mL}$ of Periciazine solution were taken with pipette and transferred to a $50 \mathrm{~mL}$ volumetric flask together with $5 \mathrm{~mL}$ of $0.2 \mathrm{~mol}$ $\mathrm{L}^{-1}$ solution of hydrochloric acid and $20.0 \mathrm{~mL}$ of a $\quad 0.005 \mathrm{~mol} \mathrm{~L}^{-1}$ potassium hydrogen peroxymonosulfate solution.Resulting solution was carefully shaked and the volume of the solution was filled to the mark with doubledistilled water. Finally, the flask was closedand mixed thoroughly. The solution was measured at an analytical wavelength of $362 \mathrm{~nm}$ in a $1 \mathrm{~cm}$ thick quartz cuvette against the double-distilled water as a compensating solution.

The content of Periciazine, in calculation on Periciazine base $\left(\mathrm{C}_{21} \mathrm{H}_{23} \mathrm{~N}_{3} \mathrm{OS}\right)$, in $\mathrm{mg}$ in one capsule, $(\mathrm{X})$ is calculated by the equation 1 :

$$
\mathrm{X}=\frac{C_{s t} \times A_{x} \times 100 \times \bar{m}}{A s t \times m}
$$

where $A_{x}$ isoptical density in an experiment with a solution of capsules,

Ast-represents an optical density of the solution in the experiment with the Periciazine WSS; $\mathrm{C}_{s t}$ iscontent of Periciazine active substance in WSS, $\mathrm{mgmL}^{-1}$; mis weight of the powder contained in the capsules, $g ; 100$ - volume of the volumetric flask, taken for the WSS or drug preparation;

$\bar{m}$ represents average weight of the capsule content, g.

Method of determining the content of Periciazine in a solution
An accurately measured volume of $0.5 \mathrm{~mL}$ of solution was transferred to a $200 \mathrm{~mL}$ volumetric flask. The solution was diluted to 200 $\mathrm{mL}$ with $50 \%$ solution of ethanol. The flask was closed and mixed thoroughly.

Using a pipette, a volume of $5.00 \mathrm{~mL}$ of resulting Periciazine solution was transferred into $50 \mathrm{~mL}$ volumetric flask with $5 \mathrm{~mL}$ of 0.1 mol L ${ }^{-1}$ solution of sulfuric acid and $10.0 \mathrm{~mL}$ of $0.005 \mathrm{~mol} \mathrm{~L}^{-1}$ potassium hydrogen peroxymonosulfate solution. Finally, the volume of the solution was filled to the mark using double-distilled water, closed and mixed thoroughly. The solution was measured at wavelength $362 \mathrm{~nm}$.

Into another $50 \mathrm{~mL}$ volumetric flask, successively $5.00 \mathrm{~mL}$ of pre-diluted (1:400) 4\% (40 $\mathrm{mg}$ to $1.00 \mathrm{~mL}$ ) Neuleptil solution, $5.00 \mathrm{~mL}$ of $0.10 \mathrm{mg} \mathrm{mL}^{-1}$ of Periciazine WSS, $5 \mathrm{~mL}$ of $0.1 \mathrm{~mol} \mathrm{~L}^{-1}$ solution of sulfuric acid and $20 \mathrm{~mL}$ of $0.005 \mathrm{~mol} \mathrm{~L}^{-1}$ potassium hydrogen peroxymonosulfate solution were mixed, bring the volume of the solution to the mark with double-distilled water, close and mix thoroughly. The solution was measured at wavelength $362 \mathrm{~nm}$.

The content of Periciazine, in calculation on Periciazine base $\left(\mathrm{C}_{21} \mathrm{H}_{23} \mathrm{~N}_{3} \mathrm{OS}\right)$ in $\mathrm{mg}$ in one capsule $(\mathrm{X})$ is calculated by theequation 2 :

$$
\mathrm{X}=\frac{C_{a} \times A_{x} \times 400}{\left(A_{x+a}-A_{x}\right)}
$$

where $A_{x+a}$ is optical density in the test with the test solution and with the solution of the Periciazine additive, $A_{x}$ - optical density in an 
experiment with Periciazine test solution, $\mathrm{C}_{a}-$ content of the Periciazine base in the solution of the additive $\mathrm{mg} / \mathrm{mL}, 400$ - dilution of $4 \%$ (40 $\mathrm{mg} / \mathrm{mL}$ ) solution of the preparation.

\section{Results and discussion}

A study of the kinetics of the reaction by the method of iodometric titration of an oxidizing agent showed that in an acidic medium (0.001-0.01 mol L $\mathrm{L}^{-1} \mathrm{HCl}$ or $\mathrm{H}_{2} \mathrm{SO}_{4}$ ) at a temperature of $15-25^{\circ} \mathrm{C}$, Periciazine is oxidized almost instantly (observation time $1 \mathrm{~min}$ ). One mole of Periciazine consumes one mole of oxidizing agent, which indicates the formation of sulfoxide. Fig. 2 shows the UV absorption spectra of S-oxidation products of Periciazine in a solution of $0.02 \mathrm{~mol} \mathrm{~L}^{-1} \mathrm{HCl}$ at a change in its concentration from 5 to $40 \mu \mathrm{gmL}^{-1}$, obtained with $1 \cdot 10^{-3} \mathrm{~mol} \mathrm{~L}^{-1} \mathrm{KHSO}_{5}$.As can be seen, an oxidation product is observed, characterized by a band with a maximum at $362 \mathrm{~nm}$. Proceeding from the literature, the chemistry of the process can be represented by the scheme (Fig. 3)

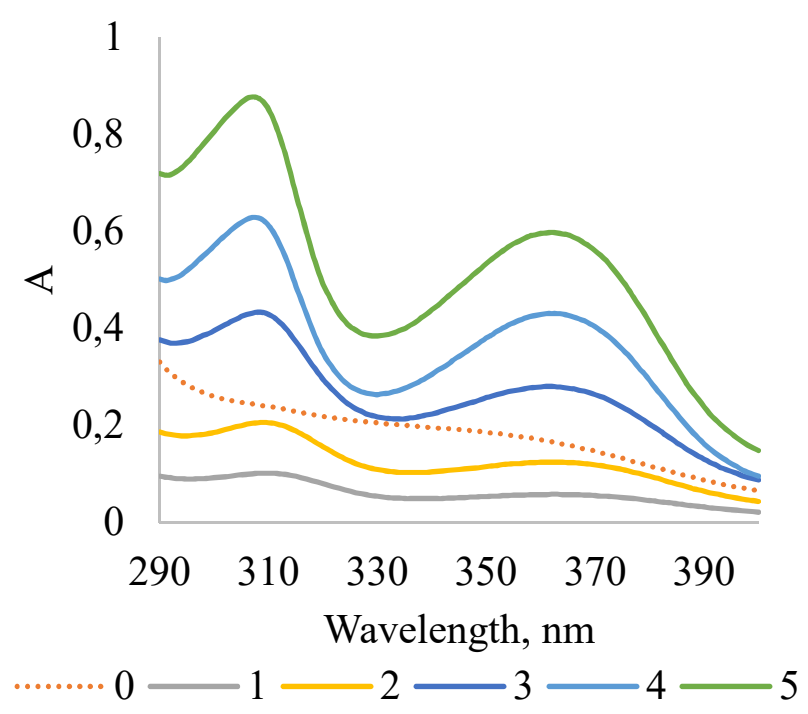

Figure 2. UV spectra of light absorption of S-oxidation product of Periciazine. $0.02 \mathrm{~mol} \mathrm{~L}^{-1} \mathrm{HCl} . \mathrm{C}, \mu \mathrm{gmL}^{-1}: 1-5$; $2-10 ; 3-20 ; 4$ to $30 ; 5-40$; UV absorption spectra of unoxidized Periciazine, 0 - 20, $\mu \mathrm{gmL}^{-1}$

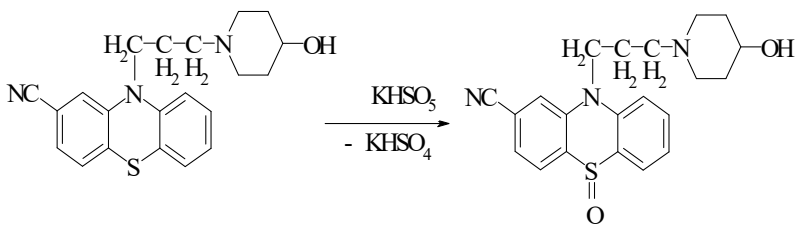

Figure 3. Scheme of S-oxidation reaction of potassium Periciazine with hydrogen peroxymonosulfate in acidic medium

The reaction product - the corresponding sulfoxide - is stable over time (the optical density at a given acidity did not change with the observation time 2 hours). The optical density of solutions at the maximum of light absorption is a linear function of the concentration of the derivative phenothiazine (the equation has the form

$A=(0.015 \pm 0.0005) \cdot C \quad$ characterized with correlation coefficient of 0.999) (Fig. 4).

According to the calibration graph, the limit of detection (LOD) and the limit of quantification (LOQ) were calculated to be $0.9 \mu \mathrm{gmL}^{-1}$ and $2.8 \mu \mathrm{gmL}^{-1}$, respectively.

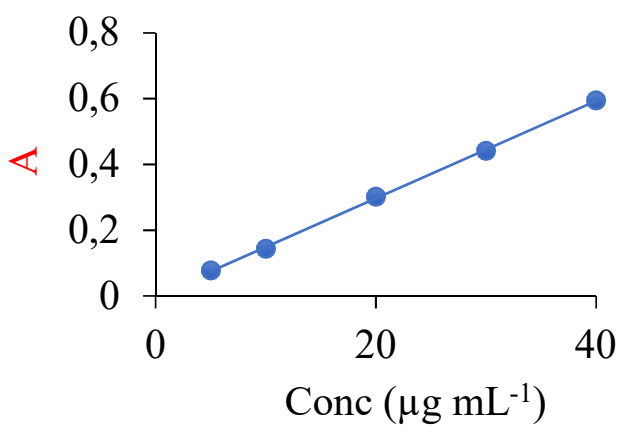

Figure 4. Concentration dependence of light absorption of oxidation product (calibration graph for quantitative determination) of Periciazine 
Tables 1 and 2 show results obtained from determination of Periciazine in $10 \mathrm{mg}$ capsules, as well as $4 \%$ solution (drops) (40 $\mathrm{mgmL}^{-1}$ ) of Periciazine using the novel methods. They testified that proposed method of analysis performance allows to determine the Periciazine in finished dosage forms with reliable accuracy. The RSD lower than $1.76 \%$ was calculated. The results are in good correspondence with the findings of the study of phenothiazine derivative in $10 \mathrm{mg}$ capsules and $40 \mathrm{mg} \mathrm{mL}^{-1}$ solutions (drops) in accordance with the recommended in European Pharmacopoeia $(\delta=<\mathrm{RSD})$.

Determination of Periciazine in $10 \mathrm{mg}$ capsules, as well as $4 \%$ solution for intravenous use in the presence of a number of inactive ingredients with a corresponding sulfoxide, obtained with potassium hydrogen peroxymonosulfate, is more sensitive, faster and less laboriousness compared to methods based on the formation of free radicals of phenothiazine, as well as a simpler than a HPLC techniquementioned in introduction. It should be noted that the technique developed by our scientific group allows to determine Periciazine in the presence of ascorbic acid without their prior separation.
Table 1. Results of Quantitative Determination of Periciazine in Neuleptil ${ }^{\circledR} 10 \mathrm{mg}$ capsules

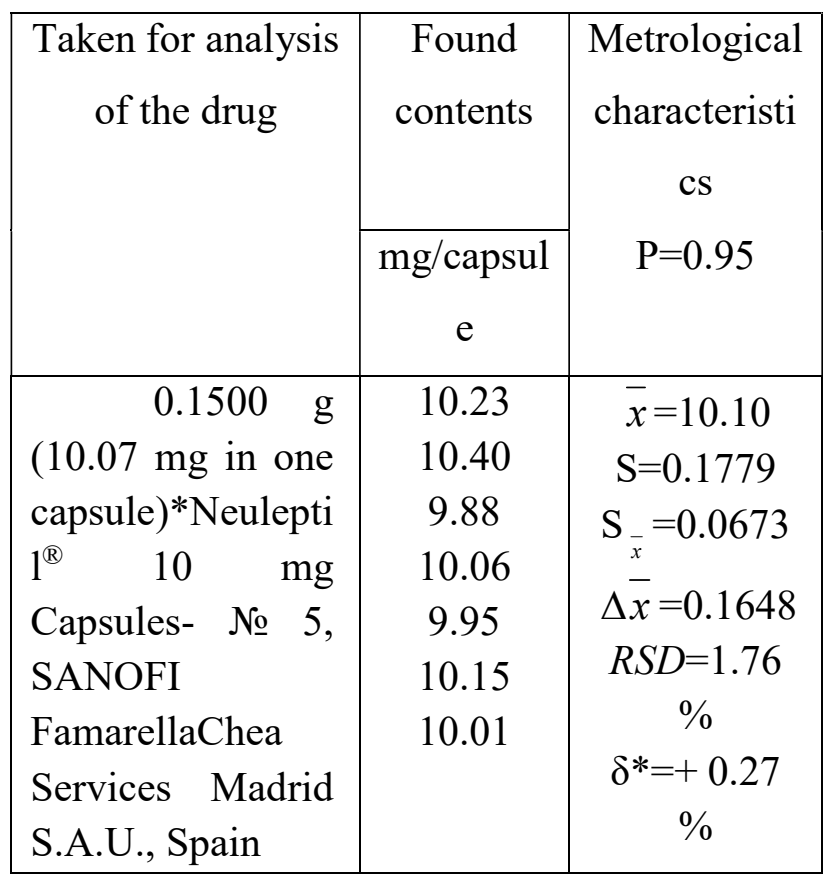

Notes: * Calculated according to the certificate of analysis; $\delta=(\bar{x}-\mathrm{a}) 100 / \mathrm{a}$; a - the content specified on the certificate.. Limits: $9.5-10.5 \mathrm{mg}$ Periciazine / 1 caps. (95 - 105\%).

Thus, for the first time, in the practice of pharmaceutical analysis the method for indirect spectrophotometric determination of 10piperidyl alkyl derivatives of phenothiazine on the example of Neuleptil in pharmaceutical preparations based on reactions of peroxyacid Soxidation was proposed. 
Table 2. Results of Quantitative Determination of

Periciazinein $30 \mathrm{~mL}$ of $4 \%$ Neuleptilsolution

Notes: * Calculated according to data on

\begin{tabular}{|c|c|c|}
\hline $\begin{array}{c}\text { Taken for } \\
\text { analysis of the } \\
\text { solution }\end{array}$ & $\begin{array}{c}\text { Found } \\
\text { contents } \\
\%\end{array}$ & $\begin{array}{c}\text { Metrological } \\
\text { characteristics } \\
\qquad \mathrm{P}=0.95\end{array}$ \\
\hline $\begin{array}{c}0.50 \mathrm{ml} \\
(3.96 \%)^{*} \text { drops } \\
\text { solution } \\
\text { SANOFI - } \\
\text { AVENTIS } \\
\text { FRANCE } \\
\text { (France), } \\
\text { produced by A. } \\
\text { Hutterman\&Sie, } \\
\text { GmbH, } \\
\text { Germany.; № } \\
\text { 6K0331 series }\end{array}$ & $\begin{array}{l}3.91 \\
3.84 \\
3.92 \\
3.88 \\
3.82 \\
3.99 \\
4.00\end{array}$ & $\begin{array}{c}\bar{x}=3.91 \\
\mathrm{~S}=0.069 \\
\mathrm{~S}_{\bar{x}}=0.026 \\
\Delta \bar{x}=0.0638 \\
R S D=1.76 \% \\
\delta^{*}=-1.13 \%\end{array}$ \\
\hline
\end{tabular}

certificate (HPLC). $\delta=(\bar{x}-\mathrm{a}) 100 / \mathrm{a}$; a - the content specified on the certificate.

\section{Conclusions}

A completely new spectrophotometric procedures were developed for quantitative determination of Periciazine in pharmaceutical preparations. The common excipients employed do not interfere in the determination of phenothiazine drug. Results of analysis of the drug dosage forms by the proposed method are in good agreement with those of the official method.

\section{References}

[1] Danilov D.S. Periciazine «forgotten» by researchers and its significance for modern clinical practice. Psychiatry and psichopharmacotherapy. 2015; 1: 3137. [In Russ.].
[2] Bekker R.A., Bykov Yu.V. Pericyazine and its clinical used. Psychiatry and Psychopharmacotherapy. 2018; 20 (2):16-31 [In Russ.].

[3] Sajjan AG, Ramesh KC, Jaldappagari Seetharamappa, Keshavayya J. Spectrophotometric investigations on the assay of phenothiazine drugs. Indian J Pharm Sci. 2002 Jun; 64(6): 603-606.

[4] Puzanowska-Tarasiewicz H, Karpińska J, Kuźmicka L. Analytical Applications of Reactions of Iron(III) and Hexacyanoferrate(III) with 2,10-Disubstituted Phenothiazines. Int $J$ Anal Chem. 2009 Feb; 2009:302696. Available from: http://www.ncbi.nlm.nih.gov/pubmed/20107561

[5] Mielech-Łukasiewicz K, Puzanowska- Tarasiewicz H, Panuszko A. Electrochemical Oxidation of Phenothiazine Derivatives at Glassy Carbon Electrodes and Their Differential Pulse and Square-wave Voltammetric Determination in Pharmaceuticals. Anal Lett. 2008 Apr; 41(5):789-805. Available from: http://www.tandfonline.com/doi/abs/10.1080/0003271 $\underline{0801935038}$

[6] Lux M, Vigneron J, Perrin A, Hoffman MA, Hoffman M. Refractometric Screening of Oral Syringes. Can. J Hosp. Pharm. 2018 Aug;48(5). Available from: https://www.cjhp-

online.ca/index.php/cjhp/article/view/2324/0

[7] Tanaka E, Nakamura T, Terada M, Shinozuka T, Hashimoto C, Kurihara K, et al. Simple and simultaneous determination for 12 phenothiazines in human serum by reversed-phase high-performance liquid chromatography. $J$ Chromatogr B. Analyt. Technol Biomed Life Sci. 2007 Jul; 854(1-2):116-20. Available from: http://www.ncbi.nlm.nih.gov/pubmed/17459789

[8] Cai HL, Deng Y, Fang PF, Cao S, Hou ZY, Wu YQ, et al. A sensitive LC-MS/MS method for analysis of pericyazine in presence of 7-hydroxypericyazine and pericyazinesulphoxide in human plasma and its application to a comparative bioequivalence study in Chinese healthy volunteers. J Pharm Biomed Anal. 
2017 Feb; 135:67-74. Available from:

https://www.sciencedirect.com/science/article/pii/S07

31708516309979?via\%3Dihub

[9] Temerdashev ZA, Kiseleva N V., Klishchenko RA, Udalov A V. Separation and identification of phenothiazine compounds by thin-layer chromatography. J Anal Chem. 2006 Jan; 61(1):2-5. Available from: http://link.springer.com/10.1134/S1061934806010023

[10] Klishchenko RA, Temerdashev ZA, Kiseleva N.V. Peculiarities of TLC screening of phenothiazine derivatives. Bulletin of universities. North Caucasian region. Natural Sciences. 2005; 3:59-62 [in Russ.].

[11] The United States Pharmacopeia, Thirty-Ninth Revision, and The National Formulary, Thirty-Fourth Edition. The United States Pharmacopeial Convention 12601 Twinbrook Parkway, Rockville, MD 20852

[12] European Pharmacopoeia $9^{\text {th }}$ Edition - European Directorate for the Quality of Medicines (EDQM) Council of Europe, 67075 Strasbourg Cedex, France 2016. 4016 p.

[13] Blazheyevskiy M.Ye. Application of derivatization by means of peroxy acid oxidation and perhydrolysis reactions in pharmaceutical analysis. Lviv: Ivan Franko National University of Lviv; 2017. 